\title{
Les lasers à cascade quantique : l'accès au moyen infrarouge
}

Les lasers à cascade quantique ont fait, depuis leur première démonstration en 1994, des progrès considérables, notamment dans le moyen infrarouge (bande allant de 3 à $12 \mu \mathrm{m}$ ). Ils peuvent maintenant être considérés comme la solution pour les applications en défense et sécurité. Les performances atteintes permettent aussi d'envisager l'intégration d'architectures photoniques plus complexes pour obtenir de nouvelles fonctionnalités en tirant profit du potentiel considérable des technologies développées autour des semi-conducteurs et de leur maturité. Nous parcourons dans cet article les progrès récents des lasers à cascade quantique, et discutons des nouvelles perspectives.

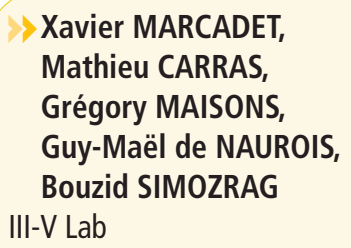

\section{Contexte et défis}

\section{Une solution de choix parmi les sources infrarouges}

La quête d'une source laser performante couvrant le moyen infrarouge (MIR - 3 à $12 \mu \mathrm{m}$ ) est toujours très vive. Plusieurs technologies sont actuellement disponibles ou en développement (figure 1). Ces sources sont les diodes laser aux sels de plomb, les sources cohérentes basées sur la génération de différence de fréquence (DFG, pour Difference Frequency Generation), les oscillateurs paramétriques optiques (OPO, pour Optical Parametric Oscillator), les lasers solides ( $\left.\mathrm{Cr}^{2+}: \mathrm{Il}-\mathrm{VI}\right)$ et les lasers à cascade quantique. Les lasers aux sels de plomb sont généralement volumineux et nécessitent un refroidissement inférieur à $90 \mathrm{~K}$. Les sources DFG, spécialement celles basées sur les cristaux de PPLN (Periodically Poled Lithium Niobate), sont matures, robustes et relativement compactes mais sont limitées à des longueurs d'onde inférieures à $5 \mu \mathrm{m}$ et à de faibles puissances optiques. Les OPOs présentent de fortes puissances crêtes mais sont cependant encore trop volumineux et coûteux pour un déploiement à grande échelle. Parmi les technologies disponibles, les sources semiconductrices apparaissent comme étant la solution à moyen terme car elles sont basées sur une seule conversion électrooptique à haut rendement. Les lasers semi-conducteurs sont de plus extrêmement compacts, fiables et peu onéreux. Les lasers à cascade quantique $(\mathrm{QCL}$, pour Quantum Cascade Laser) sont aujourd'hui

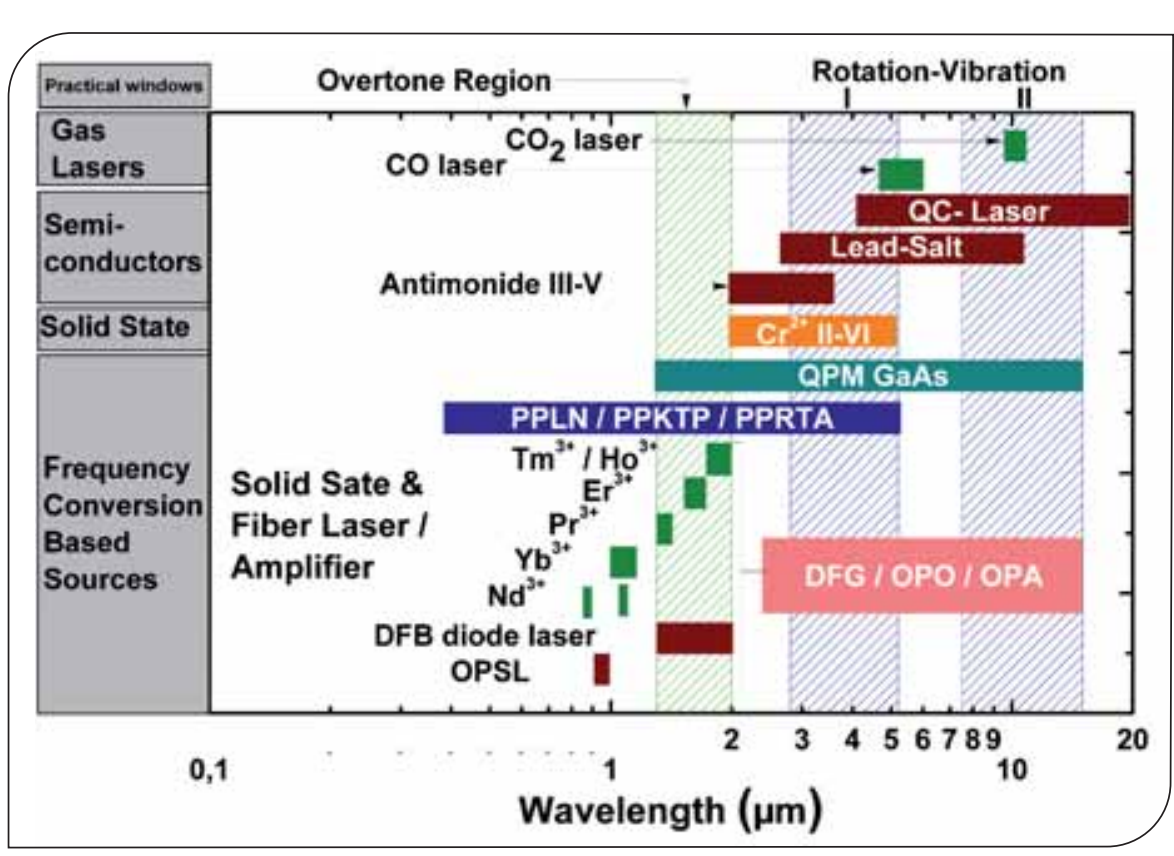

Figure 1. Différentes sources lasers dans le moyen infrarouge utilisées en spectroscopie et leurs

plages en longueur d'onde, figure tirée de la référence [1].

les seuls lasers semi-conducteurs fonctionnant à température ambiante en mode pulsé ou continu dans le moyen infrarouge.

\section{Principe du laser à cascade quantique}

Le QCL est basé sur une transition optique d'un électron entre des niveaux quantifiés de la bande de conduction [2].

Contrairement aux diodes lasers classiques, la longueur d'onde d'émission ne dépend pas directement de la bande interdite des matériaux semi-conducteurs utilisés mais principalement de l'empilement des couches formant la zone active. L'ingénierie quantique et le contrôle de l'épaisseur des couches à la couche atomique près, accessible par les techniques d'épitaxie de production (figure 2), permettent d'obtenir les niveaux d'énergie et les fonctions d'onde désirés (figure 3). Pour maximiser l'amplification optique, on juxtapose un grand nombre de zones actives, créant ainsi une structure périodique. Sous l'effet d'un champ électrique, les électrons traversent cette structure par effet tunnel puis émettent un photon 


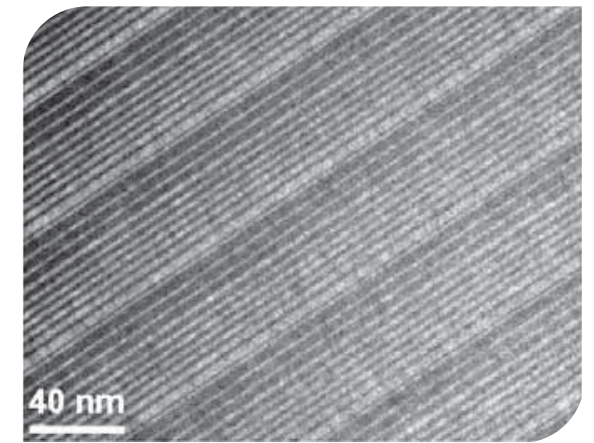

Figure 2. Photographie en microscopie électronique à transmission d'une structure périodique d'un laser à cascade quantique. Les puits quantiques sont en noir et les barrières en blanc.

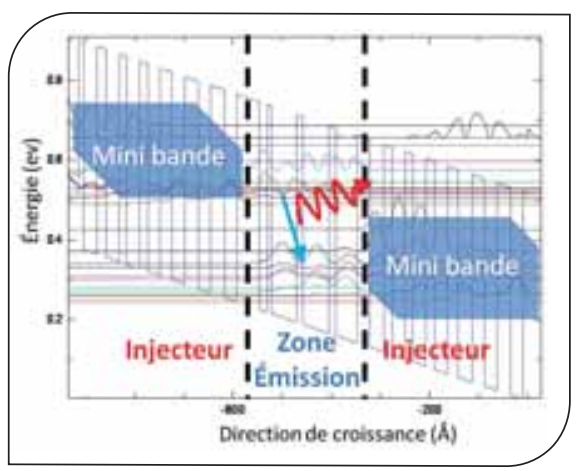

Figure 3. Diagramme typique de la bande de conduction d'un QCL montrant les niveaux d'énergie et les fonctions d'onde d'une période et demie.

et sont recyclés d'une période à l'autre. Les électrons décrivent ainsi une cascade d'énergie potentielle en émettant chacun idéalement autant de photons qu'il y a de périodes. La flexibilité unique du dessin des lasers à cascade quantique rend cette technologie à semi-conducteurs très attractive pour la fabrication de lasers sur une large plage spectrale s'étendant jusqu'au térahertz ( $\mathrm{THz})$, soit de 2,5 à $160 \mu \mathrm{m}$ [3]. Les meilleures performances sont cependant obtenues dans la bande située entre 4 et $10 \mu \mathrm{m}$. Le système de matériaux utilisés est dans ce cas le même que celui utilisé pour les diodes laser télécom (GalnAs/ AllnAs sur substrat InP).

\section{Applications et performances}

Deux familles de composants QCL peuvent être définies en fonction des applications : les sources de forte puissance, utilisées dans les systèmes de contremesures optiques pour le brouillage ou l'aveuglement des détecteurs moyen infrarouge ; les sources mono-fréquence et accordables pour la détection des explosifs, des drogues, des agents chimiques de guerre, des toxiques industriels, des agents polluants et le contrôle des procédés industriels. Pour ces deux types d'applications, une équipe de l'université de Northwestern a démontré des performances remarquables : une puissance optique de 5 Watts est obtenue pour une émission à $4,9 \mu \mathrm{m}$ pour une source multi-mode constituée d'une cavité laser de $5 \mathrm{~mm}$ de long et de $8 \mu \mathrm{m}$ de large. Le laser fonctionne en continu à température ambiante avec un rendement de conversion de $21 \%$, proche de la limite théorique de $30 \%$ [4]. Une source mono-fréquence à $4,8 \mu \mathrm{m}$ avec une puissance optique de 2,4 Watts a aussi été démontrée [5]. Ces résultats confirment le fort potentiel des lasers à cascade quantique. Pour les contre-mesures optiques et pour la détection de gaz par spectroscopie, le défi est toujours d'accroître le rendement de conversion électro-optique pour augmenter la puissance optique et/ou réduire la puissance électrique nécessaire au fonctionnement du laser. Au regard des performances actuelles, plusieurs équipes imaginent aussi la réalisation de circuits photoniques MIR intégrés pour ajouter de nouvelles fonctionnalités sur la puce semiconductrice tout en restant compact.

\section{Vers des structures innovantes pour la spectroscopie}

La plage spectrale du moyen infrarouge est particulièrement intéressante pour la spectroscopie de gaz car elle présente plusieurs fenêtres de transmission atmosphérique (avec des lignes d'absorption de $\mathrm{H}_{2} \mathrm{O}$ et $\mathrm{CO}_{2}$ faibles et peu nombreuses) où nous trouvons les raies d'absorption ro-vibrationnelles fondamentales de plusieurs polluants atmosphériques ( $\mathrm{NO}$, $\mathrm{N}_{2} \mathrm{O}, \mathrm{CO}, \mathrm{H}_{2} \mathrm{CO}$ (formaldehyde), $\mathrm{NH}_{3}$, $\mathrm{SO}_{2}$, etc). Ces raies fondamentales sont deux à trois ordres de grandeur plus intenses que leurs harmoniques dans le proche infrarouge (1 à 2,5 $\mu \mathrm{m}$ ). De plus, la plupart des molécules organiques complexes (plus de 5 atomes) qui

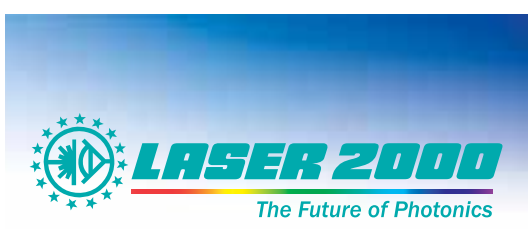

\section{Instrumentations et Mesures}

- Sphères intégrantes, Métrologie de la lumière

- Photomètre Radiomètre et Colorimètre

- Caméras hautes performances

- Monochromateurs et Spectrofluorimètres

- Mesure spectrale

- Analyseurs de faisceaux

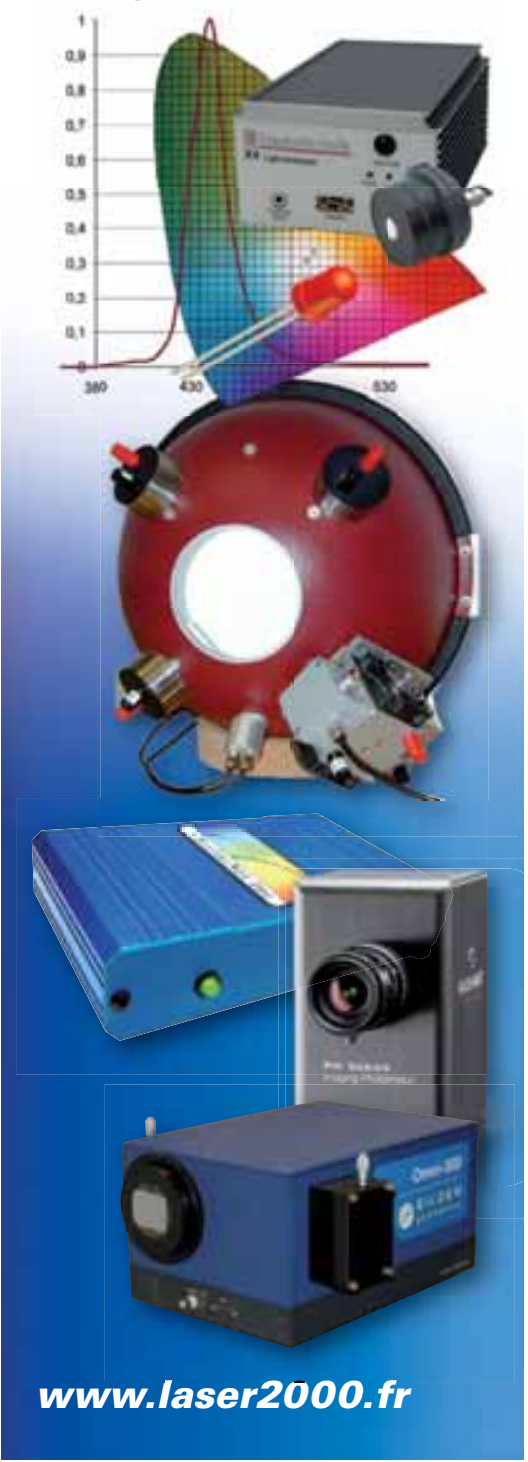


ont un intérêt pour la défense et la sécurité ont aussi leurs signatures entre 7 et $12 \mu \mathrm{m}$.

\section{Fonctionnement mono-fréquence}

Un fonctionnement mono-fréquence du laser est nécessaire pour les applications en spectroscopie. II est généralement obtenu en introduisant un réseau dans la cavité laser qui induit une contre-réaction répartie (ou DFB, pour Distributed Feed$B a c k)$. Ce réseau, qui agit comme un filtre fréquentiel, entraîne ainsi un fonctionnement mono-fréquence. Les difficultés pour dessiner et réaliser un QCL DFB ont été partiellement surmontées en utilisant des réseaux enterrés. Cependant, cette technique nécessite une étape de reprise de croissance onéreuse et critique. Une nouvelle approche basée sur l'utilisation de réseaux métalliques de surface a été développée. Cette nouvelle approche repose sur une modulation de l'indice générée par le couplage entre les modes guidés dans la zone active et un mode confiné à l'interface, entre le métal et le guide supérieur (figures 4 et 5) [6].

Précisons que ce couplage est possible car les modes contra-propagatifs d'une part, et le mode de surface d'autre part, ont la même polarisation TM. Ces réseaux métalliques de surface peuvent être utilisés dans le moyen infrarouge car les pertes sont moins élevées que dans le proche infrarouge. Un avantage supplémentaire de cette approche est sa robustesse vis-à-vis des variations technologiques, ce qui permet de contrôler la longueur d'onde d'émission à plus ou moins $1 \mathrm{~cm}^{-1}$. Des QCL DFB fonctionnant en mode pulsé ou continu sont maintenant disponibles commercialement (figure 6, voir aussi les fiches techniques sur www.3-5lab.fr) sur la totalité de la plage allant de 4 à $10 \mu \mathrm{m}$ [6]. Les lasers fonctionnent à température ambiante sur un module thermoélectrique à effet Peltier. Les puissances émises sont de quelques dizaines de milliwatts et le taux de réjection des modes latéraux (ou SMSR, pour Side Mode Suppression Ratio) est supérieur à $30 \mathrm{~dB}$. La longueur d'onde d'émission peut être accordée sur une plage de 3 à $4 \mathrm{~cm}^{-1}$ par échauffement de

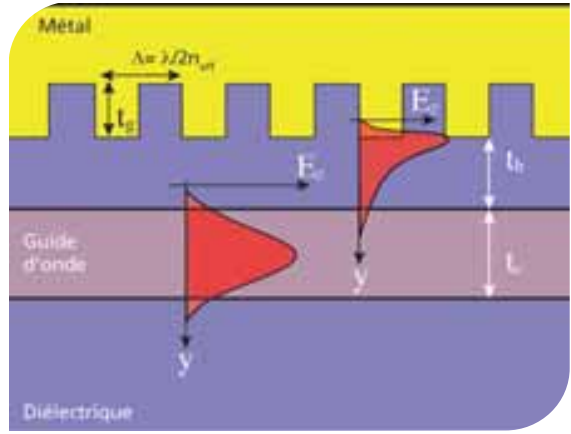

Figure 4. Schéma d'un QCL DFB avec un réseau métallique de surface. Les profils du champ dans le guide d'onde et du mode de surface avant couplage sont représentés.

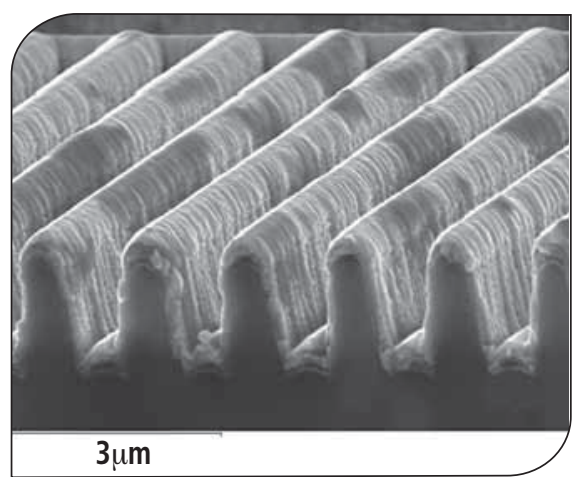

Figure 5. Photographie par microscope électronique à balayage du réseau métallique de surface d'un QCL DFB.

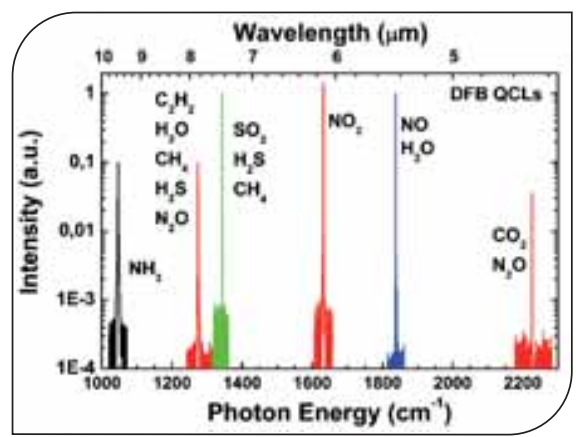

Figure 6. Spectres des différents QCL DFB. Pour chaque spectre, les molécules pour lesquelles le laser peut être utilisé en spectroscopie sont indiquées.

la structure laser en modulant le courant injecté dans le QCL DFB.

\section{QCL à émission par le substrat}

La versatilité des réseaux métalliques de surface rend aussi possible la réalisation de QCL émettant par le substrat plutôt que par la tranche. Un des avantages de cette configuration est la réduction de la divergence des QCL. En effet, les dimensions de la facette émettrice étant de

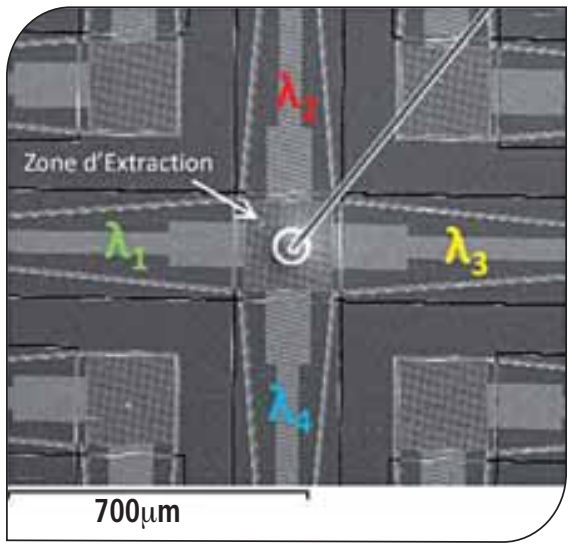

Figure 7. Photographie par microscope électronique à balayage d'un multiplexeur pour quatre QCL DFB basés sur l'utilisation d'une zone d'extraction commune utilisant un réseau métallique de surface du second ordre.

l'ordre de grandeur de la longueur d'onde d'émission, les QCL sont très divergents. En utilisant la base du guide d'onde plutôt que la facette on augmente les dimensions de la surface émettrice et on réduit ainsi la divergence. L'émission par le substrat est obtenue en utilisant des réseaux métalliques de surface du second ordre ou une combinaison astucieuse d'un réseau du premier ordre avec un réseau du second ordre [7]. La lumière dans la cavité est alors couplée vers les modes radiatifs perpendiculaires au substrat. Avec la réduction de la divergence, l'architecture optique des systèmes de spectroscopie pourrait être simplifiée et plus compacte. Cette approche permet de plus la réalisation de multiplexeurs pour les systèmes de spectroscopie multi-gaz. En effet, les fonctions de contre-réaction répartie et d'émission par la surface peuvent être séparées. On combine alors un guide DFB dont les performances ont déjà été démontrées avec une zone d'émission par la surface, le tout intégré de façon monolithique [8]. La zone d'émission peut être utilisée pour coupler plusieurs sections DFB (figure 7). On réalise ainsi une source MIR multi-longueur d'onde compacte et avec une faible divergence.

\section{Vers l'intégration de nouvelles fonctionnalités}

Tous ces développements démontrent le degré de maturité des lasers à cascade quantique pour leur utilisation dans des 


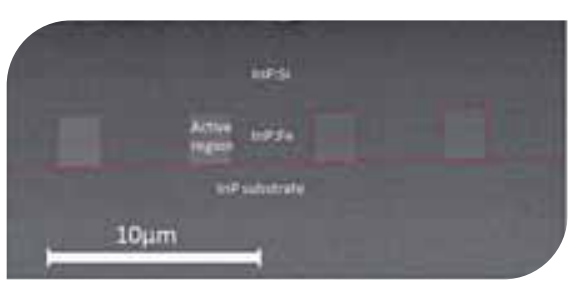

Figure 8. Photographie par microscope électronique à balayage de rubans $\mathrm{QCL}$ enterrés dans de I'InP (dopé Fe) pour la réalisation de réseaux de $\mathrm{QCL}$ cohérents.

systèmes de spectroscopie. Plusieurs PME européennes développent depuis quelques années ces systèmes. Les systèmes de spectroscopie optique actuels sont volumineux ( 20 litres, 20 kilogrammes) ce qui limite la généralisation de leur utilisation dans certains domaines d'applications (portabilité, applications grand public, ...). Dans une démarche de miniaturisation, des équipes proposent déjà l'intégration de toutes les fonctionnalités $d^{\prime}$ 'un système de spectroscopie optique sur une seule puce (lab-on-chip) par intégration hétérogène de $\mathrm{QCL}$ avec des circuits photoniques silicium [9]. On peut aussi envisager une intégration homogène en développant les circuits photoniques sur InP dans le moyen infrarouge.

\section{Des réseaux de QCL}

\section{cohérents pour les}

\section{applications de puissance}

Le domaine des QCL est aussi très actif dans le développement des sources MIR de puissance pour les systèmes de défense. Pour les systèmes de contre-mesures optiques, la source MIR doit présenter à la fois une forte puissance et une bonne qualité de faisceau. Les $\mathrm{QCL}$ répondent à ces exigences mais la technologie actuelle semble montrer ses limites, en puissance et en rendement de conversion électro-optique.

Une première solution consiste à combiner les faisceaux de plusieurs QCL en utilisant par exemple un multiplexage en longueur d'onde ou en utilisant des réseaux optiques pour la mise en phase [10]. Ces nouvelles architectures optiques peuvent être avantageuses pour de très fortes puissances et/ou pour une largeur spectrale étendue mais elles ont tendances

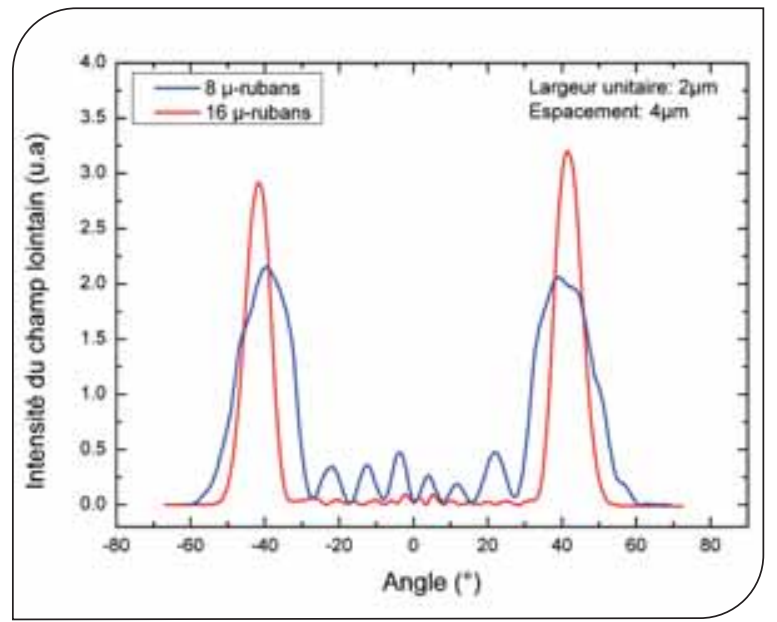

Le couplage entre les $\mu$-rubans dépend de la distance qui les sépare. Si les $\mu$-rubans sont en phase, on obtient un supermode. Puisque tous les $\mu$-rubans sont identiques, le motif du champ lointain est la figure de diffraction issue du réseau de $\mu$-rubans de taille finie. Afin de limiter le nombre de lobes principaux à un ou deux (les premiers ordres de diffraction), il est

Figure 9. Mesure du champ lointain pour des réseaux de QCL constitués de 8 et $16 \mu$-rubans de $2 \mu \mathrm{m}$ de large et espacés de $4 \mu \mathrm{m}$.

à être complexes. Une deuxième voie complémentaire est actuellement étudiée. Le principe est de combiner les faisceaux de plusieurs micro-rubans ( $\mu$-rubans) QCL issus d'une même puce et de les mettre en phase.

L'idée de réaliser un réseau de lasers cohérents a été étudiée de façon intensive dans les années 1990 pour les lasers proche infrarouge [11]. La majeure partie des inconvénients rencontrés alors peut être surmontée avec des lasers unipolaires émettant à de plus grandes longueurs d'onde, comme les QCL. Il est possible, par exemple, de séparer complètement les rubans QCL pour obtenir une meilleure stabilité du mode (figure 8). De plus, I'utilisation de plusieurs rubans étroits plutôt qu'un seul ruban large est un moyen d'améliorer les performances thermiques des QCL et donc le rendement de conversion électro-optique. En effet, la conductivité thermique de la zone active d'un QCL est faible. En diluant cette zone active dans de I'InP réalisé par reprise d'épitaxie (dopé par Fe pour le rendre semi-isolant), on décroît significativement la résistance thermique du composant en favorisant l'extraction thermique latérale via I'InP. Les réseaux de QCL cohérents intégrés de façon monolithique fournissent une solution efficace pour obtenir des lasers de puissance avec une qualité de faisceau proche de la limite de diffraction. nécessaire d'ajuster les paramètres géométriques du réseau pour éviter les ordres supérieurs de diffraction. La figure 9 présente les motifs du champ lointain obtenus pour des réseaux de 8 et $16 \mu$-rubans. Les deux motifs présentent deux lobes principaux, ce qui est caractéristique d'un supermode antisymétrique. Comme cela a été démontré dans le proche infrarouge, la qualité de faisceau des réseaux de QCL cohérents peut encore être améliorée en forçant le supermode fondamental. Cette approche permet de contourner les principales limitations vers la réalisation de $\mathrm{QCL}$ de puissances.

\section{Références}

[1] F. Tittel, D. Richter, A. Fried. Topics Applied Physics, 2003; 89: 445-516.

[2] J. Faist, F. Capasso, D. L. Sivco, C. Sirtori, A. L. Hutchinson, A. Y. Cho, Science, 1994; 264: 553.

[3] S. Barbieri, M. Ravaro, P. Gellie, G. Santarelli, C. Manquest, C. Sirtori, S. P. Khanna, E. H. Linfield, A. Giles Davies. Nature Photonics, $2011 ; 5: 306$.

[4] Y. Bai, N. Bandyopadhyay, S. Tsao, S. Slivken, M. Razeghi. Applied Physics Letters, 2011; 98: 181102.

[5] Q. Y. Lu, Y. Bai, N. Bandyopadhyay, S. Slivken, M. Razeghi. Applied Physics Letters, 2011; 98:181106.

[6] M. Carras, G. Maisons, B. Simozrag, M. Garcia, 0 . Parillaud, J. Massies, X. Marcadet. Applied Physics Letters, $2010 ; 96: 161105$.

[7] G. Maisons, M. Carras, M. Garcia, 0. Parillaud, B. Simozrag, X. Marcadet, A. De Rossi. Applied Physics Letters, 2009 ; $94: 151104$.

[8] G. Maisons, M. Carras, M. Garcia, B. Simozrag, X. Marcadet.Applied Physics Letters, $2011 ; 98: 021101$.

[9] R. Soref. SPIE Photonics West, paper 6898-5, Silicon Photonics III, 21 January 2008.

[10] G. Bloom, C. Larat, E. Lallier, M. Carras, X. Marcadet. Optics Letters, 2010; 35: 1917.

[11] D. Botez, J.C. Connolly. Applied Physics Letters $1983 ; 43: 1096$. 